\title{
Parametric Geometrical Study of OOO-Microstrip Circuit with Dual-Band Bandpass NGD Behavior
}

\author{
Rong YANG ${ }^{1}$, Fayu WAN ${ }^{1}$, Jamel NEBHEN ${ }^{2}$, Sebastien LALLECHERE ${ }^{3}$, Blaise RAVELO ${ }^{1}$ \\ ${ }^{1}$ Nanjing University of Information Science \& Technology (NUIST), Nanjing, Jiangsu, China \\ ${ }^{2}$ Prince Sattam bin Abdulaziz University, College of Computer Engineering and Sciences, \\ PO. Box: 151 Alkharj 11942, Saudi Arabia \\ ${ }^{3}$ Université Clermont Auvergne (UCA), CNRS, SIGMA Clermont, Institut Pascal, Aubière, France
}

1374903038@qq.com, fayu.wan@nuist.edu.cn,j.nebhen@psau.edu.sa, sebastien.lallechere@uca.fr, blaise.ravelo@nuist.edu.cn

Submitted October 21, 2020 / Accepted March 17, 2021

\begin{abstract}
This paper introduces a bandpass (BP) NGD circuit design engineering. The developed circuit is designed and implemented using distributed microstrip technology and operates with outstanding dual-band bandpass $N G D$ performance added with low attenuation. The BP $N G D$ topology presents an innovative geometrical shape represented by an $\mathrm{OOO}$ (triple O) structure composed of multi-parameter parallel transmission lines (TLs) based elements. The OOO type NGD circuit is mainly composed of different physical length TLs and two identical coupled lines (CLs). Then extensive parameter NGD analyses are elaborated to investigate on the influences of each physical size of $\mathrm{OOO}$ circuit on the NGD performances. Through the difference of physical length of transmission line, the delay can be adjusted. Through the results of simulation and measurement, it can be seen that the center frequency points are about $0.75 \mathrm{GHz}$ and $1.46 \mathrm{GHz}$ respectively, and the time delay is about $-1.83 \mathrm{~ns}$ and $-2.6 \mathrm{~ns}$ respectively.
\end{abstract}

\section{Keywords}

Negative group delay (NGD), bandpass NGD function, dual-band frequency, OOO-microstrip circuit, parametric analyses

\section{Introduction}

With the increase of design complexity, the delay effects due to microelectronic global delay (GD) [1] electrical interconnects [2], [3] become main phenomena limiting the electronic circuit performances. In addition to the undesirable additional noise, the GD effects cannot be currently neglected to electronic communication circuits [4], [5]. Nonetheless, some outstanding functions have been also proposed with delay lines [6], [7] and all-pass time delay circuits [8]. Behind this conventional delay function, an unfamiliar concept of negative group delay (NGD) is becoming a rising topic for $\mathrm{RF}$ and microwave research engineers by suggesting certain applications for the improvement of classical functions as the design of:

- efficiency and bandwidth enhanced feedforward amplifiers [9-11],

- asymmetrical directional coupler with NGD circuits [12],

- antenna design with NGD phase compensated serially fed array [13-15],

- recovery of RF and microwave signal integrity with NGD equalization technique [16], by reducing the delay of signal in transmission system and by avoiding signal distortion caused by delay fluctuation,

- and realization of outstanding components as non-Foster reactance with NGD circuits [15, 17, 18].

However, despite the progressive courage and efforts made by certain research groups about the NGD investigation [19-32], so far, few RF and microwave engineers understand familiarly and can develop NGD circuits for their own devices. Therefore, further research works must be performed to make the NGD circuit design to be basically familiar to non-specialists electronic, RF and microwave engineers. First, it seems that in difference with magnitude and phase, the meaning of GD is not always familiar to most of engineers. It is to be noted that as reported in [33], the GD parameter refers to the time delay generated by the signal envelope when narrowband signals pass through a linear time-invariant (LTI) transmission system. Following this magistral definition, it is important to emphasize that the NGD circuit enables to propagate peak value of output signal envelope earlier than the input one [19], [20]. Nevertheless, it was pointed out that the NGD effect must present some limitations in LTI system [21]. Further researches have been put forward about the design of higher performance as lower attenuation [22], microwave transversal filter approach design of NGD circuit [23], finite unloaded quality factor [24], compact transmission line self-matched NGD circuit [25] and simpler design parallel interconnect line [26] based NGD topologies. To alleviate the unfamiliarity of non-specialist design engi- 
neers, an innovative theory pointing out the similarity between the NGD and filter behaviors has been initiated in [27]. Extremely simple concept of low-pass, high-pass and bandpass NGD elementary functions was introduced. In the area of RF and microwave engineering, the bandpass (BP) NGD function as developed in [22-32] attracts considerably the attention of design and research engineers. In brief, it is noteworthy that because of frequency limitation, it would be interesting to find out more about the distributed circuit design feasibility without use of lumped and active components. This justifies why most of recent bandpass NGD circuits have been proposed with distributed topologies [22-32].

To continue the research works on the bandpass NGD $\mathrm{RF}$ and microwave circuit design, the design of low-loss dual-band NGD circuit is presented in this work. Among the most developed RF and microwave distributed topologies, it was found that the transmission-type coupled line (CL) based structure is more and more developed [31], [32]. However, the complexity and theoretical approach of the NGD circuit may be a major factor limiting its development. Therefore, further pedagogical study is necessary about the design engineering knowledge of BP NGD circuits. For this reason, the main interests of the present paper are to study and to describe how to design and to realize an NGD analysis of transmission line (TL) and CL based topology including different physical length elements. With dual band BP NGD circuits proposed in [28-31], the transmission attenuation is particularly severe, and affects notably the NGD performances. In difference to the research work available in [28-31], the authors investigate here on:

- a completely innovative distributed microstrip topology designed with "OOO" geometrical shapes,

- an extensive parametric analysis with respect to the physical key parameters of OOO topology,

- and low-loss multiple possibility of NGD center frequency and NGD bandwidth (BW) controls.

The present paper is composed of four different sections. Section 2 describes the BP NGD OOO-topology under study. The proof-of-concept (POC) design will be introduced with the key physical parameters of the OOO circuit. Section 3 is focused on the parametric analyses of different physical variables and illustrates how the dual-band NGD parameters are influenced. Section 4 is the experimental validation of an OOO NGD prototype with discussion between simulations and measurements. Then, Section 5 is the final conclusion.

\section{Description of the Bandpass NGD Circuit with OOO-shaped Design Geometry}

Before the detailed description of the OOO circuit, a brief definition of what was initiated BP NGD function will be introduced in a simple way, in the following paragraph.

\subsection{Bandpass (BP) NGD Function Definition}

Similar to classical RF and microwave function, the NGD analysis depends on the S-parameter representation:

$$
\mathbf{S}(s)=\left[\begin{array}{ll}
S_{11}(s) & S_{12}(s) \\
S_{21}(s) & S_{22}(s)
\end{array}\right]
$$

which assumed to model a two-port circuit by taking the Laplace variable $s=\mathrm{j} \omega$ and the angular frequency $\omega$. The associated reflection and transmission coefficient magnitudes and phase are written as, respectively:

$$
\begin{aligned}
& S_{11}(\omega)=\left|S_{11}(\mathrm{j} \omega)\right|, \\
& S_{21}(\omega)=\left|S_{21}(\mathrm{j} \omega)\right| .
\end{aligned}
$$

The associated GD is given by:

$$
G D(\omega)=\frac{-\partial \varphi(\omega)}{\partial \omega}
$$

with the phase known as:

$$
\varphi(\omega)=\arg \left[S_{21}(j \omega)\right]
$$

Under the assumption of analytical definition (4), the NGD analysis consists in exploring mathematically the existence condition of inequality:

$$
G D(\omega)<0
$$

and existence of root, $\omega=\omega_{\text {root }}$, of equations:

$$
G D\left(\omega_{\text {root }}\right)=0
$$

in function of the NGD circuit parameters. From these classical definitions, the unfamiliar BP NGD specifications [27] are defined as follows:

- The NGD center frequency denoted, $\omega_{n}$, with the equation:

$$
G D\left(\omega_{n}\right)=G D_{\min }<0
$$

- Two NGD cut-off frequencies denoted, $\omega_{1,2}$, with the equation:

$$
\left\{\begin{array}{l}
G D\left(\omega_{1,2}\right)=0 \\
\omega_{1}<\omega_{2}
\end{array}\right.
$$

- Two NGD bandwidth:

$$
B W_{\mathrm{NGD}}=\omega_{2}-\omega_{1}
$$

The ideal specifications of BP NGD function are illustrated by Figs. 1. But in difference to the classical BP filter whose bandwidth is defined by $-3 \mathrm{~dB}$ of the magnitude, the $\mathrm{BP}$ NGD circuit is defined by the GD $<0$. Inside the NGD bandwidth, $\omega_{1} \leq \omega \leq \omega_{2}$, the GD indicated by Fig. 1(a) can be supposed equal to constant: 


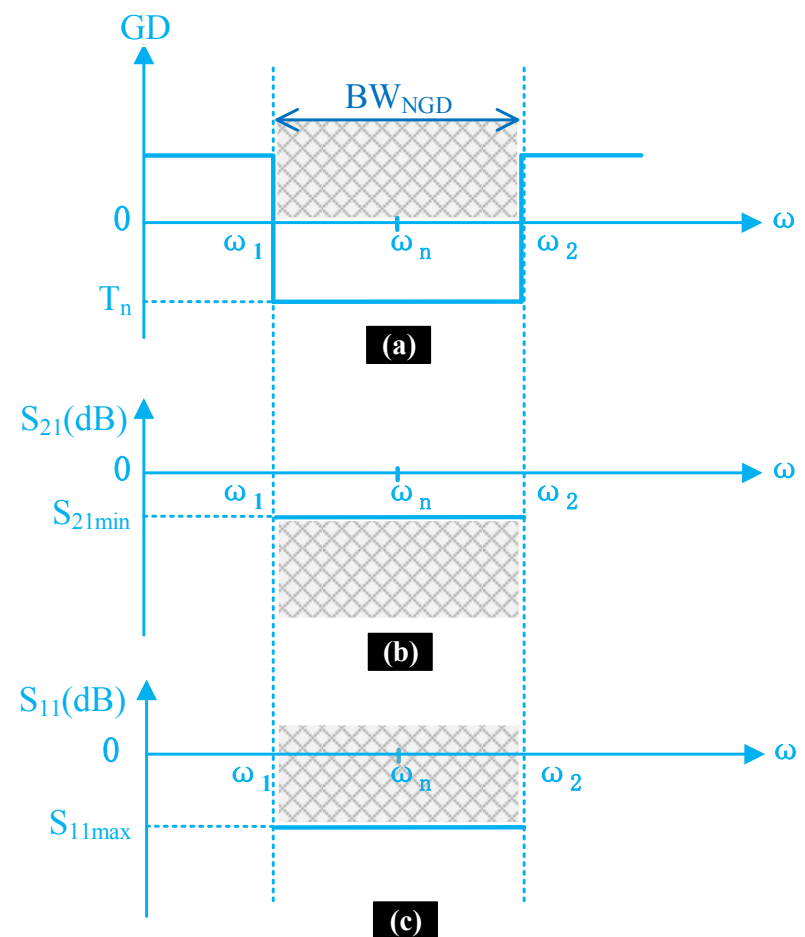

Fig. 1. (a) GD, (b) transmission and (c) reflection coefficients of ideal BP NGD function.

$$
G D(\omega)=\tau_{n}<0 .
$$

In addition to the GD response, the transmission and reflection coefficients, represented in Fig. 1(b) and in Fig. 1(c), respectively, must respect a certain performance related to low-attenuation and good access matching summarized by, respectively:

$$
\begin{gathered}
S_{21}(\omega) \geq S_{21 \text { min }}, \\
S_{11}(\omega) \leq S_{11 \text { max }}(\omega) .
\end{gathered}
$$

\subsection{Topological Analysis of OOO Circuit Diagram}

To design a typical BP NGD circuit, an OOO-shape structure was proposed. Figure 2 represents the topological diagram of this distributed circuit with any lossy lumping elements. It acts as a two-port network composed of different TLs and identical CLs. The two access ports are interconnected by parallel TLs. In Fig. 2, the left port (1) and the right port (2) serve as the input and output terminals. The circuit consists of two identical CLs and three different TLs. The later described TLs are named $\mathrm{TL}_{k}$ with $k=\{1,2,3\}$ characterized by its physical width $w$ and its physical length, $d_{k}$. In the present study, they are implemented in order to operate with characteristic impedance, $R_{0}=50 \Omega$.

Therefore, the width $w$ is chosen to be matched to $R_{0}$. This width is kept to be identical for all the TLs and CLs and determined based on the microstrip line theory [34], [35]. The CLs are represented by the physical width $w$, the

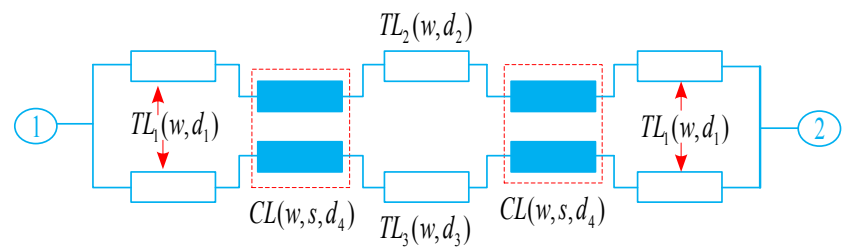

Fig. 2. Topology of the OOO NGD circuit under study.

physical space $s$, and the physical length $d_{4}$. In the next section of this study, the dual-band NGD aspect will be analyzed by illustrating the influence of physical parameters $\left(d_{k}, w, s\right)$ on:

$$
\begin{aligned}
& \left\{\begin{array}{l}
G D_{n_{1}}=\operatorname{function}\left(d_{k}, w, s\right) \\
\omega_{n_{1}}=\operatorname{function}\left(d_{k}, w, s\right) \\
B W_{\mathrm{NGD}_{1}}=\operatorname{function}\left(d_{k}, w, s\right)
\end{array}\right. \\
& \left\{\begin{array}{l}
G D_{n_{2}}=\operatorname{function}\left(d_{k}, w, s\right) \\
\omega_{n_{2}}=\operatorname{function}\left(d_{k}, w, s\right) \\
B W_{\mathrm{NGD}_{2}}=\operatorname{function}\left(d_{k}, w, s\right)
\end{array}\right.
\end{aligned}
$$

\subsection{Description of Proof-of-Concept (POC) Microstrip Circuit Design}

To realize the OOO-topology introduced in Fig. 1, the implementation in microstrip technology is considered by fixing as targeted NGD specifications:

- Dual-band NGD center frequencies around $f_{n 1}=0.75 \mathrm{GHz}$ and $f_{n 2}=1.5 \mathrm{GHz}$,

- GD value of about $G D_{n}=-2 \mathrm{~ns}$,

- Transmission coefficient better than $S_{21 \min }=-4 \mathrm{~dB}$,

- And reflection coefficient better than $S_{21 \max }=-10 \mathrm{~dB}$.

The reasons for choosing the two frequency bands of $0.75 \mathrm{GHz}$ and $1.5 \mathrm{GHz}$ are described as follows:

- The frequency bands are widely used in broadcast.

- The two frequencies are in harmonic relationship.

In fact, the proposed NGD topology operates with $f_{m}=m \cdot f_{1}$ (with for example $m=2$ or 3 or 4 ) by choosing $f_{1}$ as the fundamental NGD center frequency. The NGD values, NGD frequencies and frequency ratio, $f_{2} / f_{1}$, are sensitive and limited by:

- the substrate dispersions which are defined by the manufacturers,

- and the fabrication accuracies of the TL physical sizes and spaces.

The OOO circuit was realized on an FR4 substrate with relative dielectric constant of 4.4 , loss tangent of 0.02 , and thickness of $1.6 \mathrm{~mm}$. The substrate is metallized by copper with a thickness of $35 \mu \mathrm{m}$. Knowing these characteristics, the physical parameters of three different TLs and two identical CLs were determined. The OOO circuit was designed in order to be implemented with reduced size on 


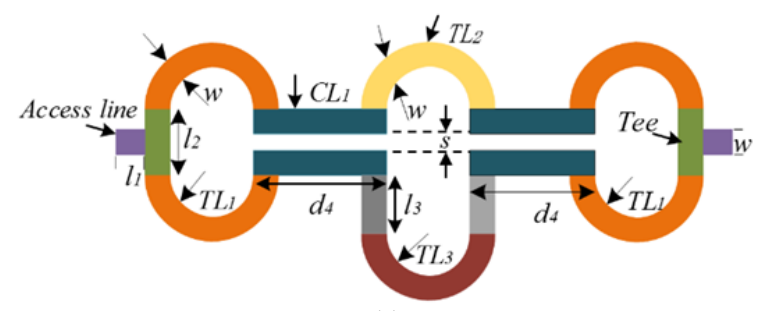

(a)

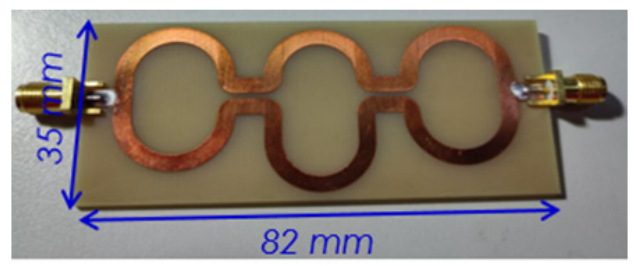

(b)

Fig. 3. OOO NGD prototype (a) layout and (b) photograph (size: $82 \mathrm{~mm} \times 35 \mathrm{~mm}$ )

\begin{tabular}{|c|c|c|c|}
\hline \multirow{2}{*}{ Elements } & $\begin{array}{c}\text { Physical } \\
\text { parameters }\end{array}$ & Name & Value (mm) \\
\hline \multirow{2}{*}{$\mathrm{TL}_{1}$} & Width & $w$ & 3 \\
\cline { 2 - 4 } & Length & $d_{1}$ & 30 \\
\hline \multirow{2}{*}{$\mathrm{TL}_{2}$} & Width & $w$ & 3 \\
\cline { 2 - 4 } & Length & $d_{2}$ & 30 \\
\hline \multirow{3}{*}{$\mathrm{TL}_{3}$} & Width & $w$ & 3 \\
\cline { 2 - 4 } & Length & $d_{3}$ & 40 \\
\cline { 2 - 4 } & Partial length & $l_{3}$ & 5 \\
\hline \multirow{3}{*}{$\mathrm{CL}_{1}$} & Width & $w$ & 3 \\
\cline { 2 - 4 } & Length & $d_{4}$ & 5 \\
\cline { 2 - 4 } & Interspace & $s$ & 3 \\
\hline \multirow{2}{*}{$\mathrm{Tee}^{*}$} & Width & $w$ & 7 \\
\cline { 2 - 4 } & Length & $l_{2}$ & 5 \\
\hline \multirow{2}{*}{ Access line } & Width & $w$ & 1 \\
\cline { 2 - 4 } & Length & $l_{1}$ & 3 \\
\hline
\end{tabular}

Tab 1. Physical parameters of OOO NGD prototype shown in Fig. 3.

a typically simple layout. Therefore, curved shape geometries were considered for certain TLs. The NGD dual-band function was generating with the adjustment of physical parameters $\left(d_{k}, w, s\right)$. As a result, the OOO circuit prototype layout shown in Fig. 3(a) was designed and implemented. The corresponding photograph is displayed in Fig. 3(b). The realized OOO prototype physical parameter values are shown in Tab. 1.

\section{NGD Parametric Analyses with Respect to the OOO-Circuit Physical Variables}

The present section is focused on the parametric analyses of the OOO circuit geometrical variable. The NGD analyses will be performed in function of physical parameter lengths $d_{k}(k=\{1,2,3,4\})$, width $w$, and space $s$ of elementary TLs and CLs. The parametric analyses are based on the S-parameters of the microstrip OOO circuit. During the analysis, the PoC circuit was simulated in the schematic environment of the RF and microwave circuit designer
ADS ${ }^{\circledR}$ commercial tool from Keysight Technologies ${ }^{\circledR}$. The simulations were performed from $0.6 \mathrm{GHz}$ to $1.6 \mathrm{GHz}$ with 801 sampling frequencies.

\subsection{Parameter Analysis with Respect to the Physical Lengths, $d_{1}, d_{2}, d_{3}$ and $d_{4}$}

The influence of $d_{k}$ has on the OOO NGD performance is investigated by simulating the S-parameter and $\mathrm{GD}$ in function of each length separately.

\subsubsection{Parameter Analysis versus $d_{1}$}

The first parametric analysis is performed with $d_{1}$ varied from $31 \mathrm{~mm}$ to $35 \mathrm{~mm}$. Accordingly, the results of S-parameters, $S_{11}, S_{21}$ and $G D$ are displayed in Fig. 4(a), (b) and (c), respectively. As expected in Fig. 4(a), the OOO circuit behaves as dual-band bandpass function for the three cases of the varied physical lengths. Moreover, Fig. 4(b) confirms that the circuit is well matched in the NGD bandwidth.

As illustrated by the zoom plots of Fig. 5(a), (b), the reflection coefficients, $S_{11}$, are better than $-10 \mathrm{~dB}$ in both NGD bandwidths. Then, Fig. 5(c), (d) reveal that the transmission coefficients, $S_{21}$, are better than $-4 \mathrm{~dB}$. It can be seen in Fig. 5(e), (f), that the first NGD center frequency point increases from $0.68 \mathrm{GHz}$ to $0.73 \mathrm{GHz}$, and the second one changes from $1.37 \mathrm{GHz}$ to $1.46 \mathrm{GHz}$. It can be emphasized from this result that the both NGD center frequencies vary inversely proportional to $d_{1}$. More importantly, it can be emphasized from this first parametric analysis that when $d_{1}$ increases, as shown in Fig. 5(e), (f), the GD value does not change significantly but the NGD center frequencies are decreasing.
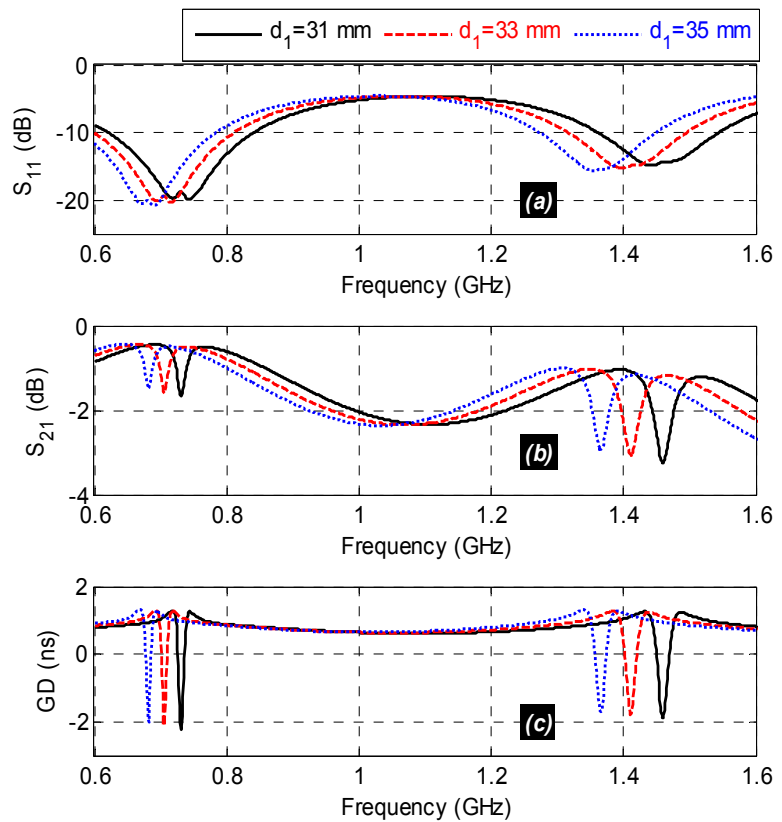

Fig. 4. (a) $S_{11}$, (b) $S_{21}$ and (c) $G D$ versus $d_{1}$. 

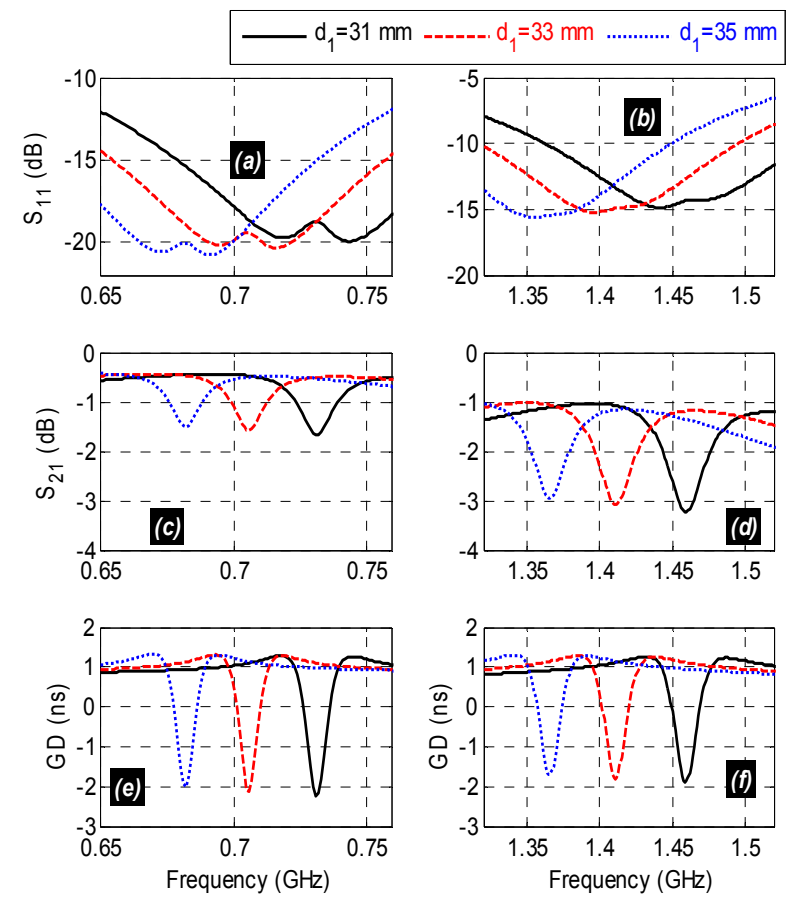

Fig. 5. (a) $S_{11}$ of $0.75 \mathrm{GHz}$, (b) $S_{11}$ of $1.46 \mathrm{GHz}$, (c) $S_{21}$ of $0.75 \mathrm{GHz}$, (d) $S_{21}$ of $1.46 \mathrm{GHz}$, (e) GD versus $d_{1}$ of $0.75 \mathrm{GHz}$ and (f) GD versus $d_{1}$ of $1.46 \mathrm{GHz}$.

\subsubsection{Parameter Analysis versus $\boldsymbol{d}_{2}$}

Figure 6 displays the parametric analysis results with respect to physical length $d_{2}$. In this case, this length was changed from $29 \mathrm{~mm}$ to $33 \mathrm{~mm}$. It can be noticed that the OOO circuit conserves its dual-band bandpass NGD behavior for all this range of $d_{2}$. It can be understood from Fig. 6(a) that the circuit is well matched in both NGD bands. Then, the attenuation loss is kept lower than $4 \mathrm{~dB}$ as shown in Fig. 6(c). It can be seen in Fig. 6(e), (f) that the first NGD center frequency is about $0.74 \mathrm{GHz}$, and the second one is at about $1.46 \mathrm{GHz}$. The NGD center frequencies are not sensitive to the change of $d_{2}$. As shown in Fig. 6(a), (b), with the increase of $d_{2}, S_{11}$ becomes smaller and varies from $-17.4 \mathrm{~dB}$ to $-25.4 \mathrm{~dB}$ and $-13.6 \mathrm{~dB}$ to $-21 \mathrm{~dB}$, respectively. As shown in Fig. 6(c) and Fig. 6(d), the increase of $d_{2}$ changes the transmission coefficient from $-2 \mathrm{~dB}$ to $-1 \mathrm{~dB}$ and $-3.8 \mathrm{~dB}$ to $-2 \mathrm{~dB}$ for the first and second NGD bands, respectively. Figure 6(e) and Figure 6(f) state that the increase of $d_{2}$ decreases considerably the NGD absolute values and they vary from $-2.92 \mathrm{~ns}$ to $-0.9 \mathrm{~ns}$ and $-2.3 \mathrm{~ns}$ to $-0.6 \mathrm{~ns}$, respectively.

\subsubsection{Parameter Analysis versus $d_{3}$}

Figure 7 depicts the broadband plots of $S_{11}, S_{21}$ and GD with respect to the parametric analysis of $d_{3}$. In this study, this length was varied from $39 \mathrm{~mm}$ to $43 \mathrm{~mm}$. It is noteworthy that the analysis results of the present case are exactly opposite to that of previous case referring to Fig. 6. Moreover, as stated in Fig. 7(a) and Fig. 7(b), the reflection coefficients at the NGD center frequencies are changing from $-21.5 \mathrm{~dB}$ to $-17 \mathrm{~dB}$ and from $-16 \mathrm{~dB}$ to $-12 \mathrm{~dB}$, for the first and second NGD bands, respectively. Moreover, as displayed in Fig. 7(c) and Fig. 7(d), the transmission coeffi- cients vary, respectively, from $-1.5 \mathrm{~dB}$ to $-2.5 \mathrm{~dB}$ and from $-2.9 \mathrm{~dB}$ to $-4.6 \mathrm{~dB}$. It can be understood in Fig. 7(e) and Fig. 7(f) that the NGD values change from $-1.8 \mathrm{~ns}$ to $-4 \mathrm{~ns}$ and from $-1.5 \mathrm{~ns}$ to $-3 \mathrm{~ns}$. Compared to the parametric analyses of $d_{1}$ and $d_{2}$, the NGD center frequencies are very less sensitive to the change of $d_{3}$.

\subsubsection{Parameter Analysis versus $d_{4}$}

Figure 8 shows that parameter analysis results with respect to length $d_{4}$ increased from $4 \mathrm{~mm}$ to $12 \mathrm{~mm}$. It can be
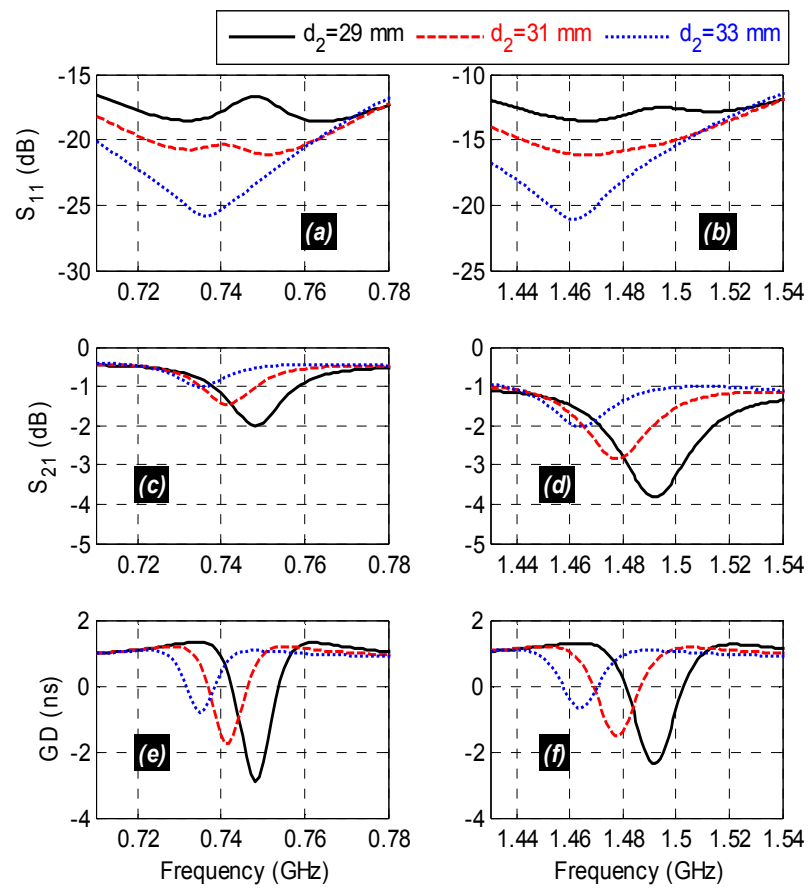

Fig. 6. (a) $S_{11}$ of $0.75 \mathrm{GHz}$, (b) $S_{11}$ of $1.46 \mathrm{GHz}$, (c) $S_{21}$ of $0.75 \mathrm{GHz}$, (d) $S_{21}$ of $1.46 \mathrm{GHz}$, (e) GD versus $d_{2}$ of $0.75 \mathrm{GHz}$ and (f) GD versus $d_{2}$ of $1.46 \mathrm{GHz}$.
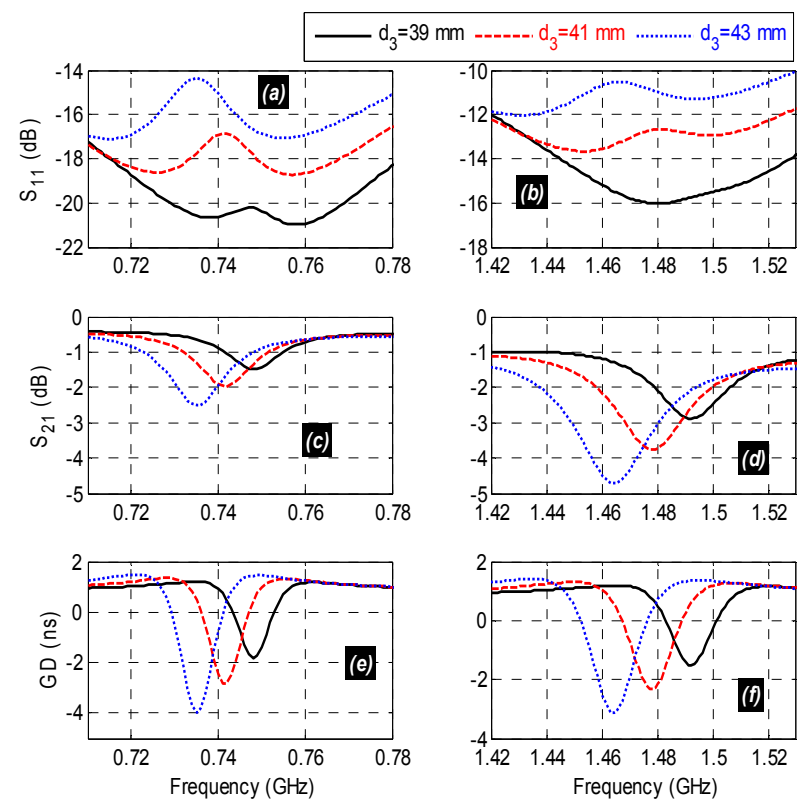

Fig. 7. (a) $S_{11}$ of $0.75 \mathrm{GHz}$, (b) $S_{11}$ of $1.46 \mathrm{GHz}$, (c) $S_{21}$ of $0.75 \mathrm{GHz}$, (d) $S_{21}$ of $1.46 \mathrm{GHz}$, (e) GD versus $d_{3}$ of $0.75 \mathrm{GHz}$ and (f) $\mathrm{GD}$ versus $d_{3}$ of $1.46 \mathrm{GHz}$. 

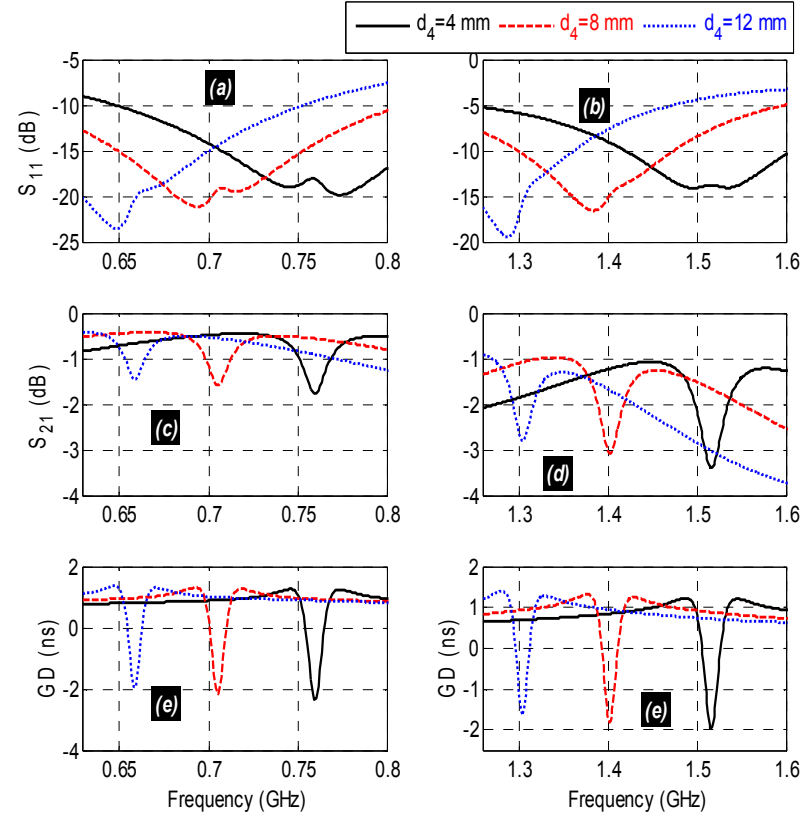

Fig. 8. (a) $S_{11}$ of $0.75 \mathrm{GHz}$, (b) $S_{11}$ of $1.46 \mathrm{GHz}$, (c) $S_{21}$ of $0.75 \mathrm{GHz}$, (d) $S_{21}$ of $1.46 \mathrm{GHz}$, (e) GD versus $d_{4}$ of $0.75 \mathrm{GHz}$ and (f) GD versus $d_{4}$ of $1.46 \mathrm{GHz}$.
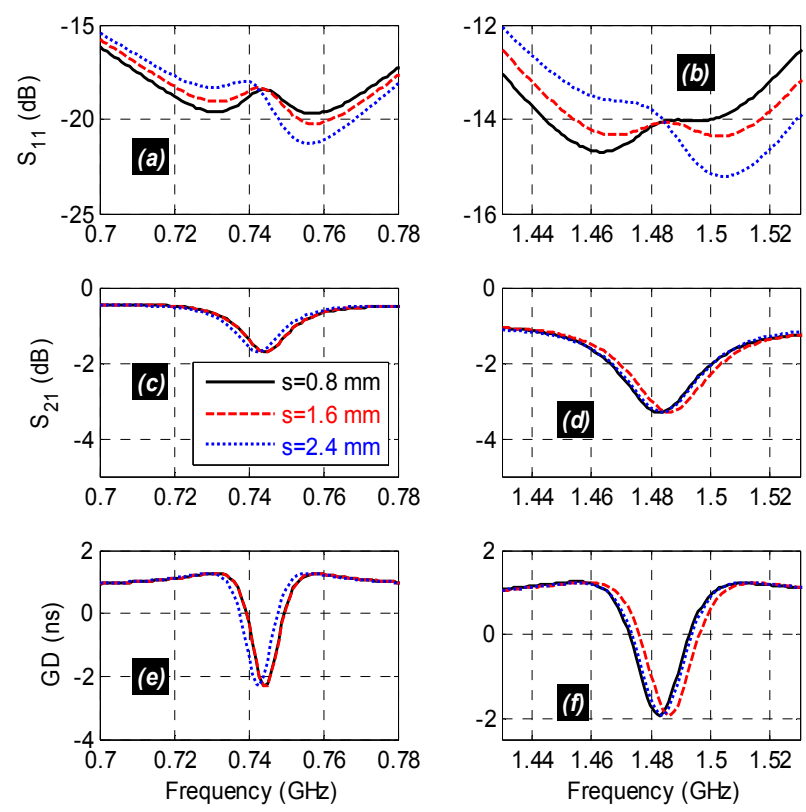

Fig. 9. (a) $S_{11}$ of $0.75 \mathrm{GHz}$, (b) $S_{11}$ of $1.46 \mathrm{GHz}$, (c) $S_{21}$ of $0.75 \mathrm{GHz}$, (d) $S_{21}$ of $1.46 \mathrm{GHz}$, (e) GD versus $s$ of $0.75 \mathrm{GHz}$ and (f) GD versus $s$ of $1.46 \mathrm{GHz}$.

seen that the increase of $d_{4}$ decreases the NGD center frequencies significantly. It can be understood from Fig. 8(a) that the OOO circuit remains well matched even $d_{4}$ is swept up. Figure 8(c) allows to underline that the two center frequency points are from $0.75 \mathrm{GHz}$ to $0.62 \mathrm{GHz}$ and from $1.54 \mathrm{GHz}$ to $1.28 \mathrm{GHz}$. As shown in Fig. 8(a) and Fig. 8(b), the reflection coefficients at the center frequencies shift from $-19 \mathrm{~dB}$ to $-23 \mathrm{~dB}$ and from $-14 \mathrm{~dB}$ to $-19.6 \mathrm{~dB}$, for the first and second NGD bands, respectively. As shown in Fig. 8(c) and Fig. 8(d), the transmission coefficients at the center frequencies change from $-1.75 \mathrm{~dB}$ to $-1.48 \mathrm{~dB}$ and
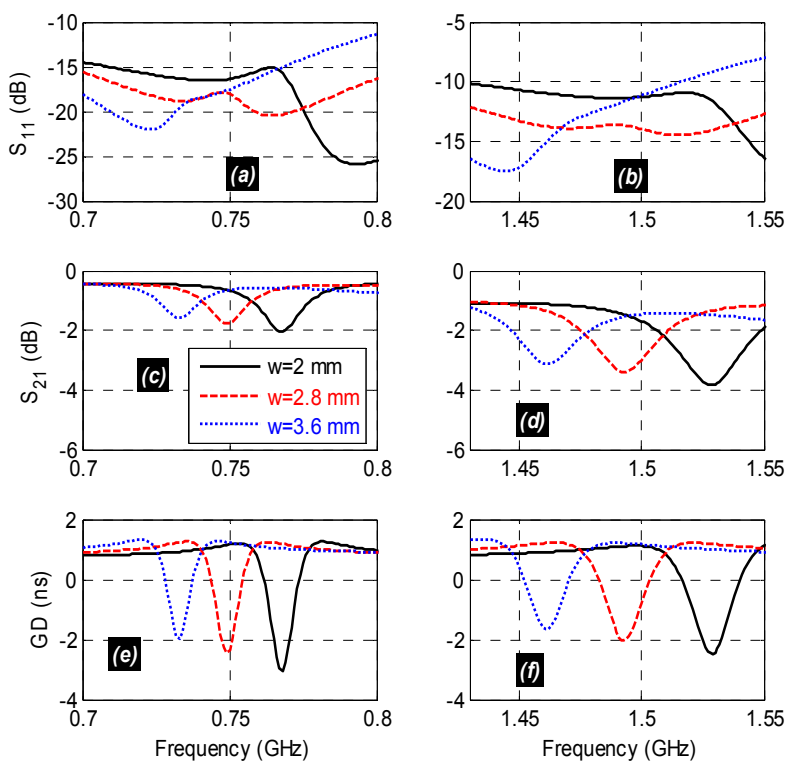

Fig. 10. (a) $S_{11}$ of $0.75 \mathrm{GHz}$, (b) $S_{11}$ of $1.46 \mathrm{GHz}$, (c) $S_{21}$ of $0.75 \mathrm{GHz}$, (d) $S_{21}$ of $1.46 \mathrm{GHz}$, (e) GD versus $w$ of $0.75 \mathrm{GHz}$ and (f) GD versus $w$ of $1.46 \mathrm{GHz}$.

$-3.4 \mathrm{~dB}$ to $-2.7 \mathrm{~dB}$. Then, the NGD values of Fig. 8(e) and Fig. $8(\mathrm{f})$ are increasing from $-2.3 \mathrm{~ns}$ to $-1.95 \mathrm{~ns}$ and $-2 \mathrm{~ns}$ to $-1.7 \mathrm{~ns}$, respectively.

\subsection{Parameter Analysis with Respect to the Space $s$}

Figure 9 presents the results of parameter analysis with respect to the coupling line $s$ varying from $0.8 \mathrm{~mm}$ to $2.4 \mathrm{~mm}$. It can be noticed that the S-parameter results are literally insensitive to the space change in the considered range of value. The NGD center frequencies are localized around $0.76 \mathrm{GHz}$ and $1.49 \mathrm{GHz}$. As illustrated in Fig. 9(a) and in Fig. 9(b), when $s$ increases, $S_{11}$ becomes smaller at both NGD center frequencies. As shown in Fig. 9(c) and Fig. 9(d), at the first and second NGD center frequencies, the changes of $S_{21}$ are not obvious. It can be emphasized from Fig. 9(e) and Fig. 9(f), the NGD values around the first and second NGD center frequencies are about $-2.25 \mathrm{~ns}$ and $-2 \mathrm{~ns}$, respectively.

\subsection{Parameter Analysis with Respect to the Physical Width $w$}

Figure 10 shows the broadband plots of OOO circuit results with respect to the change of width $w$ of the entire circuit from $2 \mathrm{~mm}$ to $3.6 \mathrm{~mm}$. The circuit remains well matched in the first NGD frequency band as illustrated by Fig. 10(a). However, the circuit becomes unmatched and $S_{11}$ is less good than $-10 \mathrm{~dB}$ in the second NGD band. As shown in Fig. 10(a), when $w=2.8 \mathrm{~mm}, S_{11}$ varies from $-20 \mathrm{~dB}$ and $-14 \mathrm{~dB}$ and when $w=2 \mathrm{~mm}$, it changes from $-25 \mathrm{~dB}$ and $-18 \mathrm{~dB}$, respectively. As shown in Fig. 10(c) and Fig. 10(d), $S_{21}$ gradually increases with the increase of $w$. At the first and second NGD center frequencies, $S_{21}$ varies from $-2 \mathrm{~dB}$ to $-1.5 \mathrm{~dB}$ and $-3.8 \mathrm{~dB}$ to $-3.1 \mathrm{~dB}$, respec- 
tively. As can be seen in Fig. 10(c), the two NGD enter frequencies are inversely proportional to the TL and CL width variations in the considered range. As illustrated in Fig. 10(e) and in Fig. 10(f), at the first and second NGD center frequencies, the GD vary from -3 ns to $-2 \mathrm{~ns}$ and -2.4 ns to $-1.6 \mathrm{~ns}$, respectively. It can be emphasized from Fig. 10(a) to Fig. 10(b), $S_{11}$ confirms that the OOO-circuit input impedance is matched very well at the NGD center frequency. The difference between $S_{11}$ is caused by the following reasons:

- Substrate material characteristic dispersion.

- Full wave simulation inaccuracies.

- Mismatch of the SMA connector to microstrip line.

- And the fabrication technology inaccuracies on the microstrip line width and thickness.

\section{Experimental Study of the OOO-Microstrip Circuit Bandpass NGD Behavior}

For the practical validation of the OOO topology, comparisons between simulated and measured results will be discussed in the present section. The present S-parameter simulation was performed with $\mathrm{ADS} \circledast$ and the measurement was done using the vector network analyzer (VNA).

\subsection{Experimental Setup Description}

Figure 11 shows a photograph of the experimental setup of the OOO circuit prototype. The experimentation was realized with a two-port VNA from Rohde \& Schwarz ${ }^{\circledR}$ ZNB20. This VNA operates in the frequency band delimited between $100 \mathrm{kHz}$ to $20 \mathrm{GHz}$. The measurement generates the touchstone S-parameters data.

\subsection{Discussion on Simulated and Experimented Dual-Band BP NGD Results}

Similar to the previous section, the present experimental results were generated in the frequency band delimited from $0.6 \mathrm{GHz}$ to $1.6 \mathrm{GHz}$. Figure 12 presents the results of ADS simulation ("Simu.") and measurement ("Meas.") in the overall frequency band. It can be seen that the simulations and measurements are in very good agreement. As expected, the simulation and measurement confirm that the OOO prototype exhibits the dual-band BP NGD behavior. The NGD dual-band center frequencies are located around $f_{n 1}=0.74 \mathrm{GHz}$ and $f_{n 2}=1.46 \mathrm{GHz}$, respectively. Table 2 summarizes the comparison between the NGD parameters. It can be pointed out that the OOO circuit presents a good NGD performance.

For further understanding about the performance in each NGD bandwidth, the zoom plots of $S_{11}, S_{21}$ and GD are shown in Fig. 12. As shown in Fig. 12(a) and Fig. 12(b), the

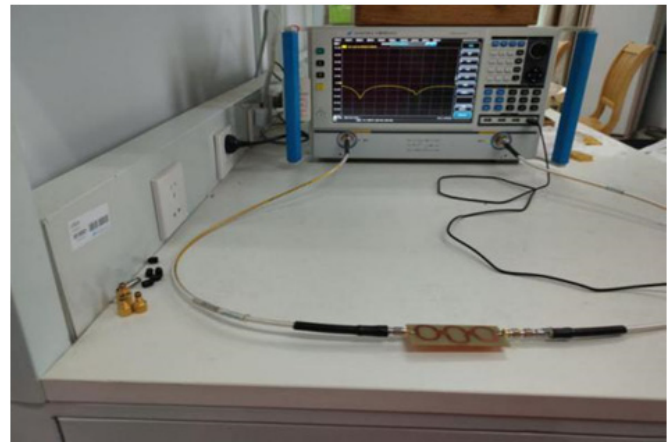

Fig. 11. OOO NGD circuit experimental setup.
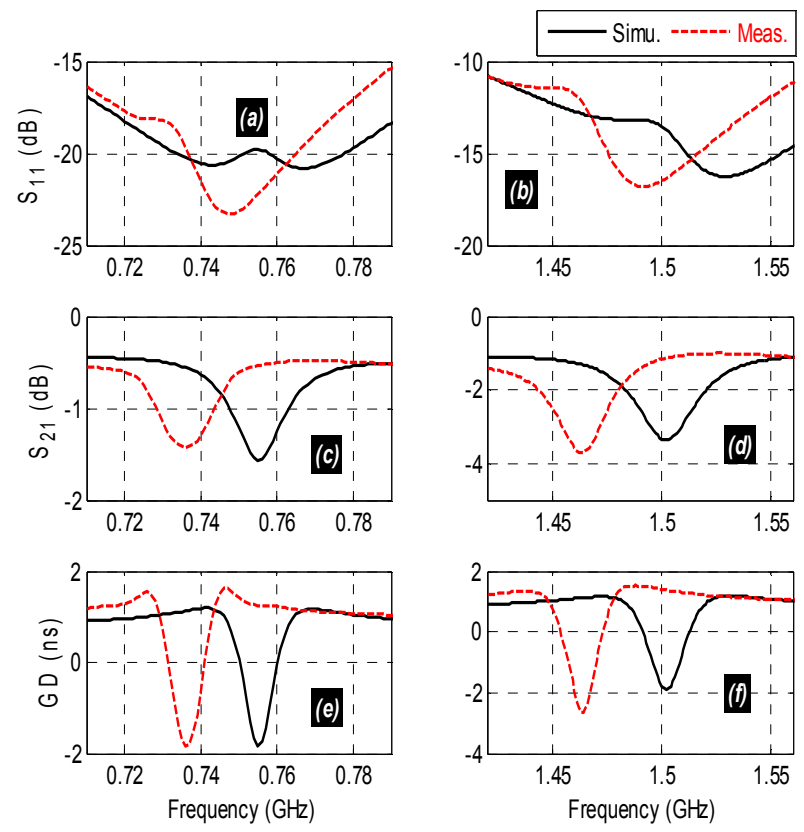

Fig. 12. Comparison between simulated (Simu.) and measured (Meas.): (a) $S_{11}$ of $0.75 \mathrm{GHz}$, (b) $S_{11}$ of $1.46 \mathrm{GHz}$, (c) $S_{21}$ of $0.75 \mathrm{GHz}$, (d) $S_{21}$ of $1.46 \mathrm{GHz}$, (e) GD of $0.75 \mathrm{GHz}$ and (f) $\mathrm{GD}$ of $1.46 \mathrm{GHz}$ from the $\mathrm{OOO}$ circuit shown in Fig. 3.

\begin{tabular}{|c|c|c|c|c|c|}
\hline $\begin{array}{c}\text { Validation } \\
\text { method }\end{array}$ & $\begin{array}{c}\text { Center } \\
\text { frequency } \\
(\mathbf{G H z})\end{array}$ & $\begin{array}{c}\boldsymbol{S}_{\mathbf{1 1}} \\
\mathbf{( d B )}\end{array}$ & $\begin{array}{c}\boldsymbol{S}_{\mathbf{2 1}} \\
(\mathbf{d B})\end{array}$ & $\begin{array}{c}\boldsymbol{B} \boldsymbol{W}_{\mathbf{N G D}} \\
(\mathbf{M H z})\end{array}$ & $\begin{array}{c}\boldsymbol{G D}_{\boldsymbol{n}} \\
(\mathbf{n s})\end{array}$ \\
\hline Simulation & 0.76 & -20.8 & -1.56 & 10 & -1.83 \\
\cline { 2 - 6 } & 1.5 & -16.3 & -3.36 & 20 & -1.9 \\
\hline Experiment & 0.74 & -23.3 & -1.43 & 8 & -1.83 \\
\cline { 2 - 6 } & 1.46 & -16.5 & -3.19 & 19 & -2.65 \\
\hline
\end{tabular}

Tab. 2. NGD specifications of the tested and simulated OOO circuit prototype.

\begin{tabular}{|c|c|c|c|c|}
\hline \multirow{2}{*}{ Ref. } & $\boldsymbol{f}(\mathbf{G H z})$ & $\boldsymbol{G D}(\mathbf{n s})$ & $\boldsymbol{S}_{\mathbf{2 1}}(\mathbf{d B})$ & $\boldsymbol{S}_{\mathbf{1 1}}(\mathbf{d B})$ \\
\hline \multirow{2}{*}[28]{} & $f_{1}=2.14$ & -3 & -34.2 & -17 \\
\cline { 2 - 5 } & $f_{2}=3.5$ & -3.1 & -34.9 & -17 \\
\hline \multirow{2}{*}[29]{} & $f_{1}=3.5$ & -4.54 & -47.4 & - \\
\cline { 2 - 5 } & $f_{2}=5.15$ & -4.2 & -38.8 & - \\
\hline \multirow{2}{*}[30]{} & $f_{1}=0.667$ & -1.19 & -18.2 & -24.8 \\
\cline { 2 - 5 } & $f_{2}=1.377$ & -1.19 & -18.2 & -24.7 \\
\hline \multirow{2}{*}{ This } & $f_{1}=0.74$ & -1.83 & -1.43 & -23.3 \\
\cline { 2 - 5 } work & $f_{2}=1.46$ & -2.65 & -3.19 & -16.5 \\
\hline
\end{tabular}

Tab. 3. BP NGD performance comparison. 
reflection coefficients are widely better than $-10 \mathrm{~dB}$ in both NGD bands. As reported by Fig. 12(c) and Fig. 12(d), the transmission coefficient is better than $-4 \mathrm{~dB}$. Then, Figures 12(e) and 12(f) confirm that the NGD bandwidth in the first band is about $10 \mathrm{MHz}$, and in the second band is about $20 \mathrm{MHz}$. For both bands, the NGD values are very close to -2 ns. Nevertheless, slight differences can be noticed between the simulations and measurements. The slight deviations of NGD values and NGD center frequencies are mainly due to the circuit manufacturing errors, the dispersions of substrate dielectric constant and loss tangent, and also the accuracy of the auxiliary calculation method.

\subsection{Discussion the BP NGD Performances of the Proposed NGD Circuit}

The BP NGD performance comparative results between the proposed NGD topology and the existing ones available in the literature [28-30] are summarized in Tab. 3. The introduced NGD topology presents the following advantages:

- Significant design simplicity,

- Considerable fully distributed elements without lossy lumped component,

- Low signal attenuation less than $3.19 \mathrm{~dB}$,

- And the reflection coefficient better than $-16.5 \mathrm{~dB}$ and without the need for external matching networks in the NGD bandwidth.

\section{Conclusion}

An investigation on NGD dual-frequency BP circuit is developed by considering an $\mathrm{OOO}$ shape microstrip structure. The OOO circuit consists of two CLs and TLs mounted as a parallel line structure. The OOO topology is built with fully distributed structure, does not need additional lumped elements.

The circuit design is described with the different geometrical parameters. In order to verify the dual-band bandpass NGD function feasibility, a microstrip circuit POC was designed, simulated, fabricated and tested. As expected, the test results confirm the characteristics of dual-band bandpass NGD function. Moreover, the OOO prototyped circuit shows that simulated and measured results are in good correlation. The OOO NGD circuit has low attenuation transmission coefficient and very good matching in the NGD bandwidths. Nevertheless, the main drawback of the OOO NGD topology concerns the second NGD center frequency is twice the first one. This dependence may limit the OOO NGD circuit usability in a real use.

In the future, the studied OOO NGD circuit can be potentially used in RF and microwave devices. As ongoing research, we expect to design dual-band tunable NGD circuits by considering an innovative non-Foster inductor and capacitor as introduced in [36] to solve the problem of delay in frequency setting and microwave circuits.

\section{References}

[1] KANG, S.-M., CHEN, H. Y. A global delay model for domino cmos circuits with application to transistor sizing. International Journal of Circuit Theory and Applications, 1990, vol. 18, no. 3, p. 289-306. DOI: 10.1002/cta.4490180306

[2] HWANG, M-E., JUNG, S-O., ROY, K. Slope interconnect effort: Gate-interconnect interdependent delay modeling for early CMOS circuit simulation. IEEE Transactions on Circuits and Systems I: Regular Papers, 2009, vol. 56, no. 7, p. 1428-1441. DOI: 10.1109/TCSI.2008.2006217

[3] RAVELO, B. Delay modelling of high-speed distributed interconnect for the signal integrity prediction. European Physical Journal Applied Physics, 2012, vol. 57, no. 3, p. 1-8. DOI: 10.1051/epjap/2012110374

[4] GROENEWOLD, G. Noise and group delay in active filters. IEEE Transactions on Circuits and Systems I: Regular Papers, 2007, vol. 54, no. 7, p. 1471-1480. DOI: 10.1109/TCSI.2007.900181

[5] MYOUNG, S.-S., KWON, B.-S., KIM, Y.-H., et al. Effect of group delay in $\mathrm{RF} \mathrm{BPF}$ on impulse radio systems. IEICE Transactions on Communications, 2007, vol. 90, no. 12, p. 3514 to 3522. DOI: $10.1093 /$ ietcom/e90-b.12.3514

[6] HEYDE, E. C. Theoretical methodology for describing active and passive recirculating delay line systems. Electronics Letters, 1995, vol. 31, no. 23, p. 2038-2039. DOI: 10.1049/el:19951356

[7] VEMAGIRI, J., CHAMARTI, A., AGARWAL, M., et al. Transmission line delay-based radio frequency identification (RFID) tag. Microwave and Optical Technology Letters, 2007, vol. 49, no. 8, p. 1900-1904. DOI: 10.1002/mop.22599

[8] WIJENAYAKE, C., XU, Y., MADANAYAKE, A., et al. RF analog beamforming fan filters using CMOS all-pass time delay approximations. IEEE Transactions on Circuits and Systems I: Regular Papers, 2012, vol. 59, no. 5, p. 1061-1073. DOI: 10.1109/TCSI.2012.2185294

[9] NOTO, H., YAMAUCHI, K., NAKAYAMA, M., et al. Negative group delay circuit for feed-forward amplifier. In Proceedings of IEEE/MTT-S International Microwave Symposium. Honolulu (HI, USA), 2007, p. 1103-1106. DOI: 10.1109/MWSYM.2007.380286

[10] CHOI, H., JEONG, Y., KIM, C. D., et al. Efficiency enhancement of feedforward amplifiers by employing a negative group-delay circuit. IEEE Transactions on Microwave Theory and Techniques, 2010, vol. 58, no. 5, p. 1116-1125. DOI: 10.1109/TMTT.2010.2045576

[11] CHOI, H., JEONG, Y., KIM, C. D., et al. Bandwidth enhancement of an analog feedback amplifier by employing a negative group delay circuit. Progress In Electromagnetics Research, 2010, vol. 105 , p. 253-272. DOI: 10.2528/PIER10041808

[12] WU, C.-T. M., GHARAVI, S., ITOH, T. Negative group delay circuit based on a multisection asymmetrical directional coupler. In Proceedings of Asia-Pacific Microwave Conference Proceedings (APMC). Seoul (Korea), 2013, p. 333-334. DOI: 10.1109/APMC.2013.6695137

[13] ALOMAR, W., MORTAZAWI, A. Elimination of beam squint in uniformly excited serially fed antenna arrays using negative group delay circuits. In Proceedings of the 2012 IEEE International Symposium on Antennas and Propagation. Chicago (IL, USA), 2012, p. 1-2. DOI: 10.1109/APS.2012.6348803

[14] TASLIMI, A., ALOMAR, W., MORTAZAWI, A. Phase compensated serially fed array using the antenna as a part of negative group delay. In Proceedings of 2015 IEEE MTT-S International Microwave Symposium. Phoenix (AZ, USA), 2015, p. 1-4. DOI: 10.1109/MWSYM.2015.7166902

[15] MIRZAEI, H., ELEFTHERIADES, G. V. Arbitrary-angle squint-free beamforming in series-fed antenna arrays using non-Foster elements synthesized by negative-group-delay 
networks. IEEE Transactions on Antennas and Propagation, 2015, vol. 63, no. 5, p. 1997-2010. DOI: 10.1109/TAP.2015.2408364

[16] RAVELO, B. Recovery of microwave-digital signal integrity with NGD circuits. Photonics and Optoelectronics, 2013, vol. 2, no. 1, p. $8-16$.

[17] MIRZAEI, H., ELEFTHERIADES, G. V. Realizing non-Foster reactances using negative-group-delay networks and applications to antennas. In Proceedings of IEEE Radio and Wireless Symposium (RWS). Newport Beach (CA, USA), 2014, p. 58-60. DOI: $10.1109 /$ RWS.2014.6830144

[18] ZHU, M., WU, C.-T. M. A tunable non-Foster T-network loaded transmission line using distributed amplifier-based reconfigurable negative group delay circuit. In Proceedings of 2018 Asia-Pacific Microwave Conference (APMC). Kyoto (Japan), 2018, p. 720 to 722. DOI: $10.23919 /$ APMC.2018.8617553

[19] MAO, H., YE, L., WANG, L. G. High fidelity of electric pulses in normal and anomalous cascaded electronic circuit systems. Results in Physics, 2019, vol. 13, p. 1-9. DOI: 10.1016/j.rinp.2019.102348

[20] WANG, J.-W., FENG, Z.-H. Time-domain nature of group delay. Chinese Physics B, 2015, vol. 24, no. 10, p. 1-5. DOI: $10.1088 / 1674-1056 / 24 / 10 / 100301$

[21] KANDIC, M., BRIDGES, G. E. Limits of negative group delay phenomenon in linear causal media. Progress In Electromagnetics Research, 2013, vol. 134, p. 227-246. DOI: 10.2528/PIER12082915

[22] CHAUDHARY, G., JEONG, Y. Low signal-attenuation negative group-delay network topologies using coupled lines. IEEE Transactions on Microwave Theory and Techniques, 2014, vol. 62, no. 10, p. 2316-2324. DOI: 10.1109/TMTT.2014.2345352

[23] WU, C.-T.-M., ITOH, T. Maximally flat negative group-delay circuit: A microwave transversal filter approach. IEEE Transactions on Microwave Theory and Techniques, 2014, vol. 62, no. 6, p. 1330-1342,. DOI: 10.1109/TMTT.2014.2320220

[24] CHAUDHARY, G., JEONG, Y. Negative group delay phenomenon analysis using finite unloaded quality factor resonators. Progress In Electromagnetics Research, 2016, vol. 156, p. 55-62. DOI: 10.2528/PIER16041111

[25] WAN, F., LI, N., RAVELO, B., et al. S-parameter model of three parallel interconnect lines generating negative group-delay effect. IEEE Access, 2018, vol. 6, p. 57152-57159 DOI: 10.1109/ACCESS.2018.2872732

[26] SHAO, T., WANG, Z., FANG, S., et al. A compact transmission line self-matched negative group delay microwave circuit. IEEE Access, 2017, vol. 5, p. 22836-22843. DOI: 10.1109/ACCESS.2017.2761890

[27] RAVELO, B. Similitude between the NGD function and filter gain behaviours. International Journal of Circuit Theory and Applications, 2014, vol. 42, no. 10, p. 1016-1032. DOI: 10.1002/cta.1902

[28] CHOI, H., JEONG, Y., LIM, J., et al. A novel design for a dual-band negative group delay circuit. IEEE Microwave and Wireless Components Letters, 2011, vol. 21, no. 1, p. 19-21. DOI: 10.1109/LMWC.2010.2089675

[29] CHAUDHARY, G., JEONG, Y., LIM, J. Miniaturized dual-band negative group delay circuit using dual-plane defected structures. IEEE Microwave and Wireless Components Letters, 2014, vol. 24, no. 8, p. 521-523. DOI: 10.1109/LMWC.2014.2322445

[30] SHAO, T., FANG, S., WANG, Z., et al. A compact dual-band negative group delay microwave circuit. Radioengineering, 2018, vol. 27 , no. 4, p. 1070-1076. DOI: $10.13164 /$ re.2018.1070

[31] DAS, R., ZHANG, Q., LIU, H. Lossy coupling matrix synthesis approach for the realization of negative group delay response. IEEE Access, 2017, vol. 6, p. 1916-1926. DOI: 10.1109/ACCESS.2017.2780888
[32] CHAUDHARY, G., JEONG, Y. Transmission-type negative group delay networks using coupled line doublet structure. IET Microwaves, Antennas \& Propagation, 2015, vol. 9, no. 8, p. 748-754. DOI: $10.1049 /$ iet-map.2014.0351

[33] POZAR, D. Microwave Engineering. 4th ed. New York: Wiley, 2011. ISBN: 978-0-470-63155-3

[34] EUDES, T., RAVELO, B., LOUIS, A. Transient response characterization of the high-speed interconnection RLCG-model for the signal integrity analysis. Progress In Electromagnetics Research, 2011, vol. 112, p. 183-197. DOI: 10.2528/PIER 10111805

[35] RAVELO, B. Behavioral model of symmetrical multi-level T-tree interconnects. Progress In Electromagnetics Research B, 2012, vol. 41, p. 23-50. DOI: 10.2528/PIERB12040205

[36] ZHU, M., WU, C.-T. M. A tunable series negative capacitor using distributed amplifier-based reconfigurable negative group delay circuit. In Proceedings of the 48th European Microwave Conference (EuMC). Madrid (Spain), 2018, p. 616-619. DOI: 10.23919/EuMC.2018.8541404

\section{About the Authors ...}

Rong YANG received the B.Sc. degree in Electronic Information Engineering from Jiangsu University Jingjiang College, Zhenjiang, China, in 2019. She is currently pursuing the M.S. degree with the Nanjing University of Information Science and Technology, Nanjing, China. Her research interests include abnormal wave propagation in dispersive media and microwave circuits.

Fayu WAN received the Ph.D. degree in Electronic Engineering from the University of Rouen, Rouen, France, in 2011. From 2011 to 2013, he was a Postdoctoral Fellow with the Electromagnetic Compatibility Laboratory, Missouri University of Science and Technology, Rolla. He is currently a Full Professor with the Nanjing University of Information Science and Technology, Nanjing, China. His current research interests include negative group delay circuits, electrostatic discharge, electro-magnetic compatibility, and advanced RF measurement.

Jamel NEBHEN received the M.Sc. in Microelectronics from the National Engineering School of Sfax, Tunisia in 2007, and the Ph.D. degree from the Aix-Marseille University, France, in 2012, all in Microelectronics. From 2012 to 2018, he worked as a Postdoctoral Researcher in France in LIRMM-Lab Montpellier, IM2NP-Lab Marseille, ISEP Paris, LE2I-Lab Dijon, Lab-Sticc Telecom Bretagne Brest, and IEMN-Lab Lille. Since 2019, he joined the Prince Sattam bin Abdulaziz University in Alkharj, Saudi Arabia, as an Assistant Professor. His research interests are mainly in the design of analog and RF integrated circuits, IoT, biomedical circuit, and sensors instrumentation.

Sébastien LALLÉCHÈRE was born in Nevers, France, in 1979. He received the M.Sc. and $\mathrm{PhD}$. degrees respectively in Computational Modeling and Electronics/ Electromagnetism from Polytech Clermont and Universite Blaise Pascal, Clermont-Ferrand, France, in 2002 and 2006. He served as a Research Engineer in LASMEA, ClermontFerrand, France, in 2007 focusing on intensive computa- 
tional methods for electromagnetics. He is currently an Associate Professor at Institut Pascal and Universite Clermont Auvergne, Clermont-Ferrand, France. His research interests cover the fields of electromagnetic compatibility including antennas and propagation, complex and reverberating electromagnetic environments, electromagnetic coupling, computational electromagnetics, stochastic modeling and sensitivity analysis in electrical engineering.

Blaise RAVELO received the Ph.D. degree from the University of Brest, in 2008, and his dissertation led to research Habilitation à Diriger des Recherches (HDR) from the University of Rouen, in 2012. He is currently a Full Professor with the Nanjing University of Information Science and Technology. He is a pioneer of the negative group delay (NGD) RF/analog and digital circuits, and systems. $\mathrm{He}$ is coauthor of more than 350 articles and regularly involved in national/international research projects. He co-supervised and directed 11 Ph.D. students and 8 Ph.D. candidates who defended. He participates regularly in large Research and Development international projects. His current publication H-index is 22 (Reference: Google Scholar 2021). His research interests include microwave circuit design, electromagnetic compatibility (EMC) and interference (EMI), and signal and power integrity (SI/PI) engineering. Dr. Ravelo is the Scientific Chair of the 5th International Conference on Electromagnetic Near Field Characterization and Imaging, in 2011, a member of Advanced Electromagnetic Symposium 2013-2018 technical committee and a member of the IEEE RADIO 2015 scientific committee. He has been a URSI member and regularly invited to review articles submitted to international journals (the IEEE Transactions on Microwave Theory and Techniques, IEEE Access, IEEE Transactions on Circuits and Systems, IEEE Transactions on Electromagnetic Compatibility, IEEE Transactions on Instrumentation and Measurement, IEEE Transactions on Industrial Electronics, Journal of Electromagnetic Waves and Applications, IET Circuits, Devices \& Systems, IET Microwaves, Antennas \& Propagation, and International Journal of Electronics, etc.) and international books (Wiley, Intech Science, etc.). 